\title{
Recognizing elderly women with sleep problems and falls from information on comorbidites and medications in a continuous use
}

\author{
Ivana Kokić \\ Health Center Osijek, P.K.P.Krešimira IV, 6, Osijek, Croatia \\ Vjenceslav Martinek \\ Family Medicine Office Sanja Bekić, \\ N.Š.Zrinskog 3, Višnjevac, Croatia
}

\begin{abstract}
Sanja Bekić
Department of Internal medicine and Family medicine Faculty of Medicine, University Josip Juraj Strossmayer Osijek, Croatia

Family Medicine Office Sanja Bekić, N.Š.Zrinskog 3, Višnjevac, Croatia

Mile Volarić

Department of Internal medicine and Family medicine Faculty of Medicine, University Josip Juraj Strossmayer Osijek, Croatia

\section{Ljiljana Majnarić Trtica}

Department of Internal medicine and Family medicine Faculty of Medicine, University Josip Juraj Strossmayer Osijek, Croatia

Department of Public Health, Faculty of Dental Medicine and Health, University Josip Juraj Strossmayer Osijek, Croatia
\end{abstract}

\begin{abstract}
Geriatric syndromes represent new, comprehensive concepts, which can help identifying vulnerable elderly people with multimorbidity. There is also a problem in using the result in a general practice working environment. We suggest fast audit as a method for conducting research on a small scale. The aim of this study was to find out the specific characteristics that can be used for a fast recognition of elderly women who have sleep problems and are susceptible to falls. We conducted a survey on a sample of 105 elderly women (age range $60-80$ years, average $68.7 \pm 5.1 \mathrm{SD}$ ), in a general practice setting, in the town of Osijek, eastern Croatia. The sleep disturbance was identified using the standard Pittsburgh Sleep Quality Index, while information on falls were taken from electronic health records (eHRs) and patient interviews. We used a multiitem questionnaire for socio-demographic characteristics and lifestyle habits. EHRs were used to diagnose problems associated with sleep disturbances and falls, as well as for the use of medication that affect mental functions. We found out that $74.2 \%$ of examined women had sleep problems, and $25.7 \%$ of them experienced falls in the past two years. Diagnoses that distinguished women with and those without sleep problems included dyspepsia and urinary tract infections (50\% vs. $23.1 \%)$. Women with sleep problems, compared to those without them, more commonly used two or more medications, usually antidepressants, analgesics and anxiolytics. Lower back pain and osteoporosis were used as screening tools for women susceptible to falls, compared to women without reports of falling. (81.5\% vs $61.5 \%$ and $22.2 \%$ vs. $5.1 \%)$. Women with falls had a higher degree of overlapping with sleep disorders (81.5\%). Anxiolytics application could be a link between sleep disorders and falls (the overlapping ratio $68 \%)$.
\end{abstract}

Key words: geriatric syndromes, women, sleep disorders, falls, fast audit, general practice 


\section{FUNDING}

The authors of this publication declare that this study was supported by the grant No. VIF2105-MEFOS-13 from the University JJ Strossmayer Osijek, Croatia.

\section{INTRODUCTION}

The identification of elderly people who have an increased risk of adverse health outcomes is not easy to perform, as they are characterised by multiple chronic medical conditions (termed multimorbidity) (Salive, 2013). It has been accepted that multimorbidity may not be an inherent part of the aging process, but is prescribed to unfavourable socio-economic and psycho-social factors, such as low income, chronic psychological stress, low physical activity, poor nutrition and social isolation (Kaplan,1987). More comprehensive approaches, termed geriatric syndromes, which stand beyond the traditional disease-based classification, are now used to define these elderly persons (Onder, 2015). These syndromes, including e.g. sleep disorders, cognitive function decline, incontinentio, functional disabilities and susceptibility to falls, are known to facilitate the development of each other and of chronic comorbid conditions (van Schoor, 2002). Because these syndromes overlap, sharing common risk factors, disease patterns and underlying mechanisms, they form the basis for new, comprehensive approaches, that can be used for the identification of vulnerable elderly people (Thomas, 2017).

Sleep disturbances and the susceptibility to falls are such two geriatric syndromes that are considered to be consequences of both age-related medical conditions and medication use, as well as unfavourable psychosocial factors (Berg, 1997; Minner, 2016). Sleep disturbance is known as the driving mechanism for the development of many comorbid conditions and undesirable health outcomes (Koyanagi, 2014). Falls are one of the most disabling conditions, which lead to the premature development of immobility and the need for nursing home care (Tinetti, 1994; Vellas, 1997). Recent epidemiologic studies have revealed the major risk factors and comorbid conditions, associated with these two geriatric syndromes (Itani, 2016; Tromp, 2001). The problem, in a general practice setting, is that evidence from the large-scale studies are difficult to translate to a population that a particular general practitioner is caring for, beause this population is limited in size and has its own specific characteristics, related to the socio-economic factors, age structure and comorbidities distribution (Tinetti, 1988; Cappucio, 2010). There are also differences among the general practitioners in prescribing medication. In addition, general practitioners need fast screening methods for recognizing patients who have an increased risk of unfavourable health outcomes. They need to know how to identify the target population for screening and for a possible intervention. The age criterium, typical symptoms, diagnoses of diseases, and the list of medications in continuous use are the labelling tools.

\section{Data and the sample}

\section{METHODS}

The study was conducted in 2016 in two general medicine practices located at the same work place, in the town of Osijek, eastern Croatia. The study lasted for three months. Women 60-80 years of age (average $68.7 \pm 5.1 \mathrm{SD}$ ), who came to the practice for other reasons, were included in the study. They all gave their written informed consent. The number of participants was 105, encompassing approximately the third of the total number of elderly women registered on the patients' list. They were examined for sleep disturbances, using the standard Pittsburg Sleep Quality Index, and for falls they experienced in the last two years, using data from the electronic health records (eHRs), complemented with the patients' interview. Information on difficulties with walking were also collected, based on observation and the participants' subjective perception of this problem. The data on the number and types of diagnoses of 
chronic diseases and on continuous or frequent use of medications (at least, in the last three months) known to have the effects on mental functions, were used from eHRs.

\section{The study design}

A multi-item questionnaire was used to obtain information on: 1) socio-demographic and lifestyle habits, including education, living conditions, family issues, physical activities, a schedule of adverse affects, daily resting periods, smoking and alcohol consumption habits, 2) self-perceived health conditions, including: general health, chronic pain, mood oscillations, cognitive functions, symptoms such as sudden weakness/loss of stability/drop attacks, feellings of dizzines/vertigo, visual and hearing disorders and self-perceived changes (during the last year) in activities of daily living and weight.

\section{Statistical methods}

Categorical parameters were presented with absolute and relative frequencies. Differences in distributions were tested using the chi-square test or Fischer's exact test, where applicable. The level of significance of $\mathrm{p}<0,05$ was considered statistically significant. Results which were significant or important were presented graphically as bar diagrams.

\section{RESULTS}

Fig. 1

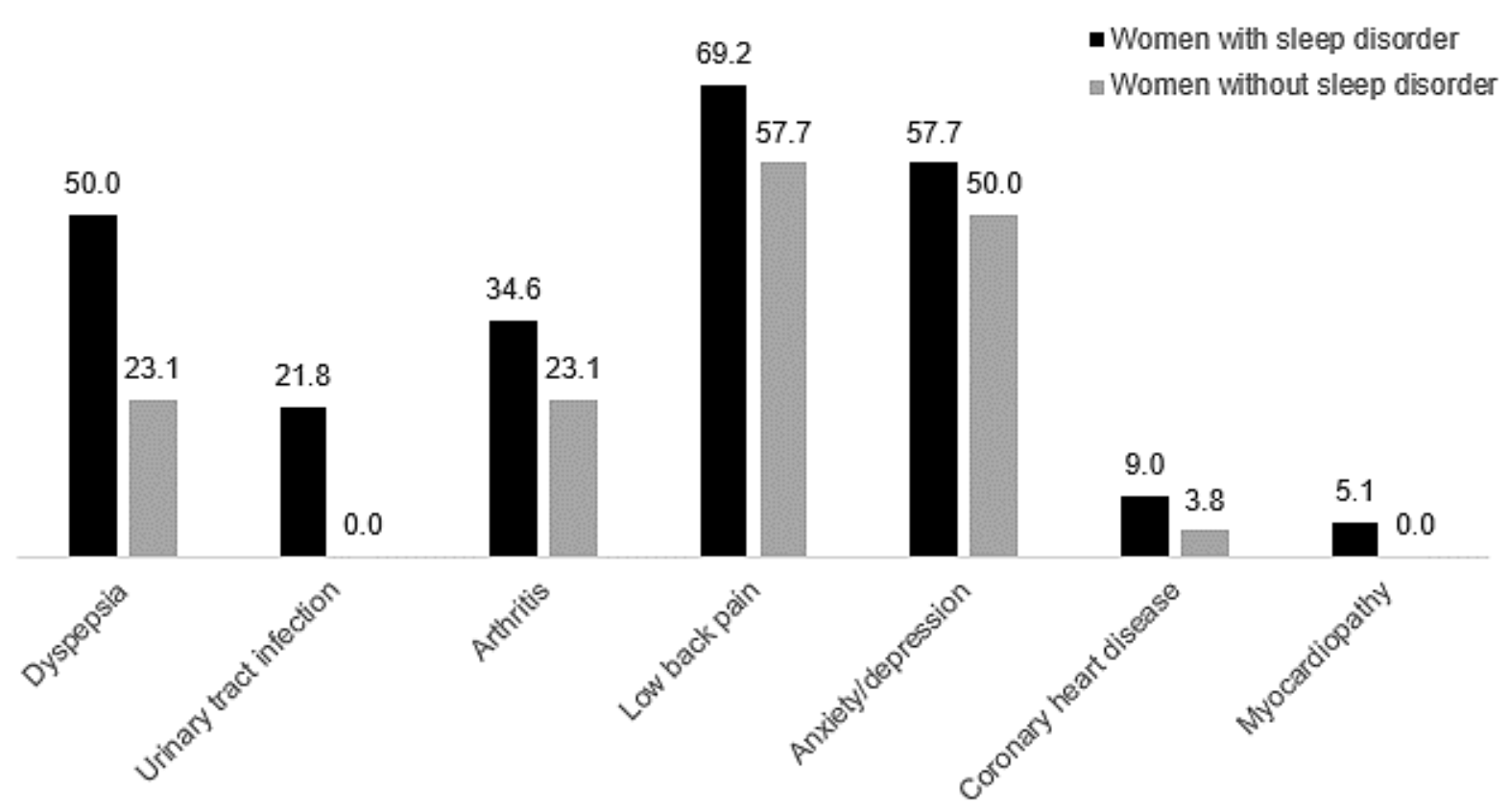

Fig. 1. The distribution ratios (percentages) of evaluated diagnoses of chronic diseases that showed significant or marked differences between elderly women with and without sleep disorders

Significant or marked differences, between elderly women who had and had not sleep disorders, were found in distribution ratios of the diagnoses indicating: dyspepsia (50\% vs $23.1 \%, \mathrm{p}=0.022)$, urinary tract infections $(21.8 \%$ vs $0 \%, \mathrm{p}=0.006)$, arthritis $(34.6 \%$ vs $23.1 \%$, $\mathrm{p}=0.336)$, low back pain (69.2\% vs $57.7 \%, \mathrm{p}=0.340)$, anxiety/depression $(57.7 \%$ vs $50 \%$, $\mathrm{p}=0.504)$, coronary heart disease ( $9 \%$ vs $3.8 \%, \mathrm{p}=0.0 .676)$ and myocardiopathy $(5.1 \%$ vs $0 \%$, $\mathrm{p}=0.570)$. 
The distribution ratios (percentages) of evaluated diagnoses of chronic diseases that showed significant or marked differences between elderly women with and without sleep disorders

Significant or marked differences, between elderly women who had and had not sleep disorders, were found in distribution ratios of the diagnoses indicating: dyspepsia $150 \%$ vs $23.1 \%, \mathrm{p}=0.022)$, urinary tract infections $(21.8 \%$ vs $0 \%, \mathrm{p}=0.006)$, arthritis $(34.6 \%$ vs $23.1 \%$, $\mathrm{p}=0.336)$, lower back pain $(69.2 \%$ vs $57.7 \%, \mathrm{p}=0.340)$, anxiety/depression $(57.7 \%$ vs $50 \%$, $\mathrm{p}=0.504)$, coronary heart disease $(9 \%$ vs $3.8 \%, \mathrm{p}=0.0 .676)$ and myocardiopathy $(5.1 \%$ vs $0 \%$, $\mathrm{p}=0.570$ ).

Fig. 2

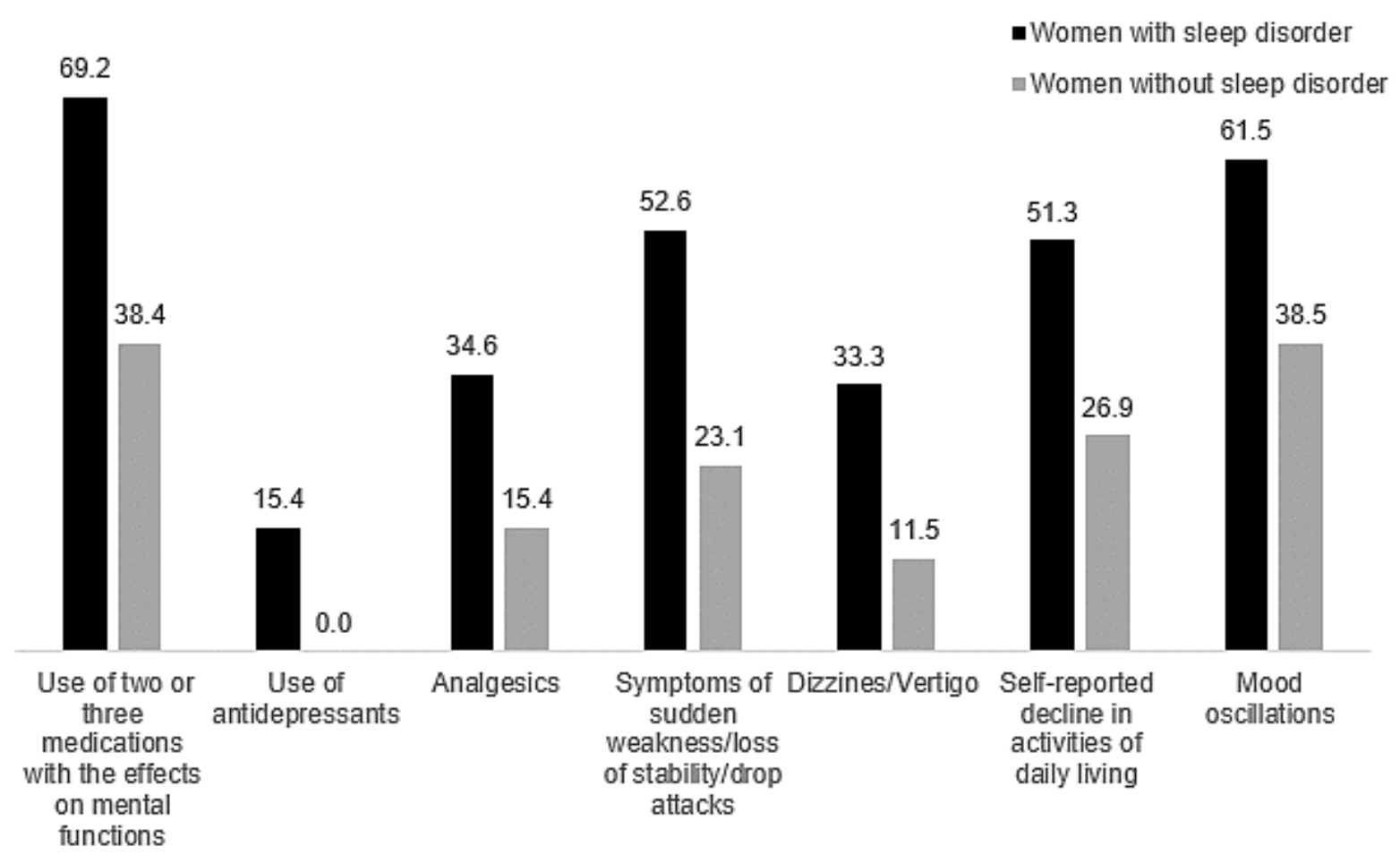

Fig. 2. The distribution ratios (percentages) of parameters indicating symptoms and medications use that showed significant or marked differences between elderly women with and without sleep disorders

Significant or marked differences, between elderly women who had and had not sleep disorders, were found in distribution ratios of parameters indicating: use of two or three medications with the effects on mental functions $(69.2 \%$ vs $38.4 \%, \mathrm{p}=0.044)$, use of antidepressants (15.4\% vs $0 \%, \mathrm{p}=0.035)$ and analgesics ( $34.6 \%$ vs $15.4 \%, \mathrm{p}=0.084$ ), symptoms of sudden weakness/loss of stability/drop attacks (52.6\% vs 23.1\%, p=0.012) and of dizzines/vertigo (33.3\% vs $11.5 \%$, $\mathrm{p}=0.042$ ), self-reported decline in activities of daily living (51.3\% vs $26.9 \%, \mathrm{p}=0.041)$ and $\operatorname{mood}$ oscillations ( $61.5 \%$ vs $38.5 \%, \mathrm{p}=0.048)$.

The distribution ratios (percentages) of parameters indicating symptoms and medication use that showed significant or marked differences between elderly women with and without sleep disorders

Significant or marked differences between elderly women with and without sleep disorders, were found in distribution ratios of parameters indicating: use of two or three medications with an effect on mental functions (69.2\% vs $38.4 \%, \mathrm{p}=0.044)$, use of antidepressants $(15.4 \%$ 
vs $0 \%, \mathrm{p}=0.035)$ and analgesics $(34.6 \%$ vs $15.4 \%, \mathrm{p}=0.084)$, symptoms of sudden weakness/loss of stability/drop attacks (52.6\% vs $23.1 \%, \mathrm{p}=0.012$ ) and of dizzines/vertigo (33.3\% vs $11.5 \%, p=0.042)$, self-reported decline in activities of daily living $(51.3 \%$ vs $26.9 \%$, $\mathrm{p}=0.041)$ and mood oscillations $(61.5 \%$ vs $38.5 \%, \mathrm{p}=0.048)$.

Fig. 3

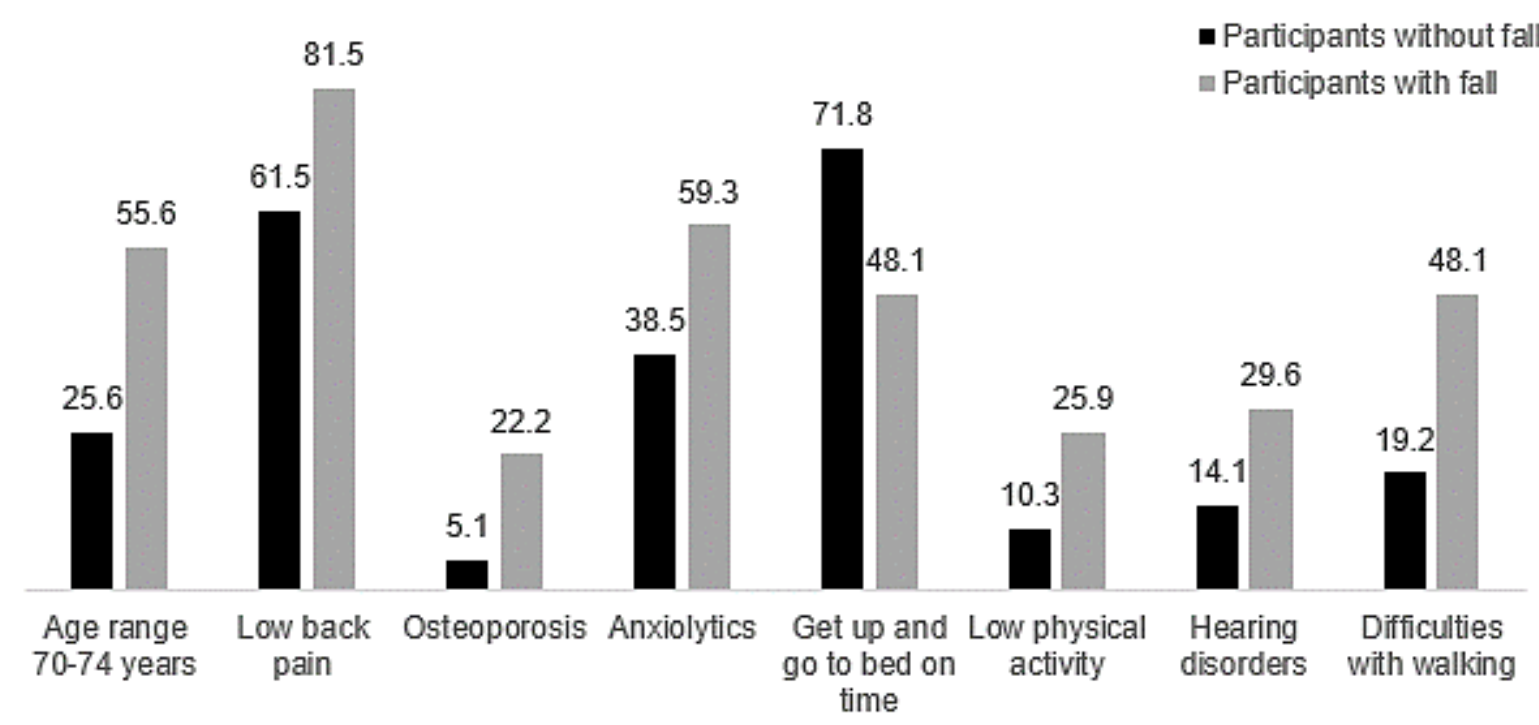

Fig. 3. The distribution ratios (percentages) of features showed significant or marked differences between elderly women with and without falls

Significant or marked differences, between elderly women who had and had not falls, were found in the distribution ratios of the parameters indicating: age range 70-74 years (55.6\% vs $25.6 \%, p=0.041)$, diagnoses of the low back pain $(p=0.063)$ and osteoporosis $(p=0.017)$, use of anxiolytics $(p=0.074)$, get up and go to bad time $(p=0.035)$, low physical activity $(p=0.058)$, hearing difficulties $(\mathrm{p}=0.086)$ and walking difficulties $(\mathrm{p}=0.005)$.

The distribution ratios (percentages) of features which showed significant or marked differences between elderly women with and without falls

Significant or marked differences between elderly women with and without falls, were found in the distribution ratios of the parameters indicating: age range $70-74$ years $(55.6 \%$ vs $25.6 \%$, $\mathrm{p}=0.041)$, diagnoses of lower back pain $(\mathrm{p}=0.063)$ and osteoporosis $(\mathrm{p}=0.017)$, use of anxiolytics $(\mathrm{p}=0.074)$, getting up and going to bed on time $(\mathrm{p}=0.035)$, low physical activity $(p=0.058)$, hearing difficulties $(p=0.086)$ and walking difficulties $(p=0.005)$.

\section{DISCUSSION}

\section{Results related to sleep disturbances}

According to the known fact that sleep disturbances are common among elderly people, especially women, our results showed a high percentage of women with sleep disorders (78 out of 105 or $74.2 \%$ ). This percentage, however, exceeded the data presented in large epidemiologic studies (Itani, 2016; Miner, 2016). We considered this finding consequential to the high multimorbidity rate, illustrated by the results showing that $80 \%$ of women with sleep disorders had 4 and more diagnoses and $50 \%$ of these women had more than 4 out of a total of 13 diagnoses of chronic diseases, which are used for evaluation and are associated with sleep disorders or with falls.These diagnosis include: hypertension, diabetes, coronary heart disease, myocardiopathy, stroke, chronic pulmonary disease, dyspepsia, arthritis, lower back pain, osteoporosis, urinary tract infections, anxiety/depression and Parkinson`s disease (Buxton, 
2012; Cappucio, 2011; Shan, 2015; Silva, 2007; Spiegel, 2009; Wang, 2015). An important fact that needs to be addressed when using diagnoses to identify elderly women with sleep disorders in a primary health care setting, is the frequency of these diseases in a small size sample, that are of interest for evaluation. For example, although cardiovascular and chronic respiratory disease are referred, in large scale studies, of being strongly associated with sleep disorders, these comorbidites, in our sample, had small occurence rates, thus leaving the major part of women with sleep disorders uncovered (Fig. 1).

There was a different situation with the diagnosis of hypertension. This condition is also widely associated with sleep disorders, but in our study, there were no differences in distribution rates of this diagnosis between women with and without sleep disorders $75.6 \%$ vs $69.2 \%$, respectively). This discrepancy was probably due to the high prevalence of hypertension, which can be found in the older population, when hypertension is studied as a reason for sleep disorders. Our results indicated some other diagnoses, which can be used for rapid identification of elderly women with sleep disorders. These diagnoses included arthritis, lower back pain and anxiety/depression which have a high occurance rate among women with sleep disorders, compared to those without these disorders $(34.6 \%, 69.2 \%$ and $57.7 \%$, respectively). Another factor are the high prescription rates of medications, such as anxiolytics, antidepressants and analgesics (prescribing rates were $47.4 \%$ vs $30.8 \%, 15.4 \%$ vs $0 \%$ and $34.6 \%$ vs $15.4 \%$, respectively) (Fig. 2).

What was interesting is that all women who used antidepressants also had sleep disturbances (Fig. 2). This finding shows high occurence rates of undifferentiated symptoms among women with sleep disorders, compared to those who did not have these disorders, indicating possible effect of medications, which may, in addition to comorbidities, negatively influence the quality of sleep in elderly women. These undifferentieted symptoms included the group of interrelated symptoms, such as sudden weakness/loss of stability/drop attacks and feelings of dizzines/vertigo ( $52.6 \%$ vs $23.1 \%$ and $33.3 \%$ vs $11.5 \%$, respectively, when comparing women with and without sleep disorders), as well as self-reported decline in activities of daily living and mood oscillations ( $51.3 \%$ vs $26.9 \%$ and $61.5 \%$ vs $38.5 \%$, in women with and without sleep disorders, respectively) (Fig. 2). This overwhelming medicalisation and high prevalence of sleep disorders among elderly women in our work environment is also supported by the result showing that the significantly higher portion of women with sleep disorders, compared to those without sleep disorders, used 2 or more medications which affect mental functions (69.2\% vs 38.4\%, respectively) (Fig.2). Practical implications of these results are that nonpharmacological measures in elderly women should replace the overwhelming use of medications.

Some other diagnoses should recieve special attention when detecting elderly women with sleep disorders, including dyspepsia and urinary tract infections, due to their high occurence rates $(50 \%$ vs $23.1 \%$ and $21.8 \%$ vs $0 \%$, respectively, when comparing women with and without sleep disorders) (Fig. 1).

\section{Results related to falls and walking difficulties}

Falls experienced in the past two years, were registered in 27 out of 105 examined women $(25.7 \%)$. This frequency is lower than it has been reported elsewhere for the general elderly population (Masud, 2001; Rubenstein, 2006). The reason for this discrepancy may be the fact that not many women with falls had significant cognitive function decline, a feature which has been generally accepted as an important risk factor for falls (Laird, 2001; Montero-Odaso, 2009; Yardley, 2002). Although a lot of examined women reported self-perceived decline in memory functions in the last year $(43.8 \%$ of a total of women in the sample and $44.4 \%$ of 
women with falls), it is not accurate enough to capture the significant shift in cognitive decline of women in the sample. The lack of the cognitive function measurement can be considered a shortcoming of this study and is recommended for future work (Sheridan, 2007). In relation to sleep disturbances, when one wants to identify the link between diagnoses of chronically diseased patients and a geriatric syndrome, the crucial thing is the frequency of a particular diagnoses in the real sample. Related to falls, this is the case with the diagnosis of Parkinson`s disease. Although evidence suggest that falls are a common problem in patients with Parkinson`s disease, this diagnosis cannot be used as a screening tool for falls in elderly women in our work environment, as there was only one such recorded case in this diagnosis (Wood, 2002).

As these results suggest, women with an increased susceptibility to falls should be looked for among women aged $70-74$ years (55.6\% of all women with falls) and among those who reported walking difficulties (ratios were $48.1 \%$ vs $19.2 \%$, for those with vs without falls). Difficulties with walking, known to predispose older women to falls (Liu-Ambrose, 2009; van Iersel, 2008), can be linked to a concrete diagnoses, including the diagnoses of lower back pain and osteoporosis, due to the fact that both diagnoses showed significant differences in distribution ratios between women with and without falls $(81.6 \%$ vs $61.5 \%$ and $22.2 \%$ vs $5.1 \%$, respectively). According to the results, these diagnoses are associated with lower back pain dysfunctions, low physical capability and frailty (Buffa, 2011; Cruz-Jentoft, 2010) with higer ratios of self-reported low physical activity found among women with falls, compared to those without falls (25.9\% vs $10.3 \%)$.

Another important entry, when recognising women susceptible to falls, could be the use of anxiolytics (Leipzig, 1999). This is indicated in the results by significant differences in anxiolytics usage ratios between women with and without falls (59.3\% vs 38.5\%). Since anxiolytics were shown to be widely used among women in the sample, as well in the whole sample (43.8\%) in women with sleep disorders (47.4\%), we performed the short-cut post hoc analysis and estimated the degree of overlaping between women who experienced falls, those who had sleep problems and those who used anxiolytics. This simple estimation showed that 22 out of 27 women with falls also had sleep problems (81.5\%), this high degree of overlaping thus indicated sleep problems as the main risk factor for falls (Winkelman, 2015). Fifteen out of 22 women also used anxiolytics (the overlap ratio was 68\%), the result indicating anxiolytics use as the factor that links sleep problems with the susceptibility to falls.

The chain of cause and effect linking these two geriatric syndromes together, can probably be explained in the following way. The disrupted sleep schedules (supported by higher rates of the self-reported disruption of getting up and going to bed in women with falls, compared to those without falls) (Fig. 3), due to chronic pain, caused by the locomotor system disease (supported by the result that $72.7 \%$ of women who had sleep problems and falls, also reported lower back pain), leads to subsequent anxiolytics use which can, together with walking difficulties (caused by the lower back segment dysfunction), lead to an increased risks of falls (Fig. 3). The opposite is also true: women who experienced falls are more likely to use anxiolytics, due to stress caused by the limitations in mobility and fear of falls. The effect of anxiolytics, as well as the stress process by itself, appears to be in the background of the ordinary sleep schedule disruption, driving the vicious cycle further on.

Well-functioning sensory organs, especially vision, are known to be a prerequisite for a stable and safe gait performance (Lord, 2010). Our results emphasized the hearing deficiency as being more prominently expressed in women with falls, compared to those who did not experience falls, representing the significant contributing factor to falls. 
Kokić, I., Martinek, V., Bekić, S., Volarić, M., \& Trtica, L. M. (2019). Recognizing elderly women with sleep problems and falls from information on comorbidites and medications in a continuous use. Advances in Social Sciences Research Journal, 6(9) 1-9.

\section{CONCLUSIONS}

The problem of connecting large-scale studies with the local situation of a particular general practice workplace, is the need for adapting the results to small size populations, which are characterised by a limited and specific scope of traits. In addition, there is a requirement that screening tools used in general practices are simple and fast to use, and specifically tailored to the selected patients groups. We suggest fast audits as a method for managing small size samples. Concerning this, we examined a part of elderly female patients, regular visitors in two general practices, placed at the same location, using a multi-item questionnaire and information from eHRs. We proved that by using this approach it is possible to find out the most prevalent medical and socio-demographic profiles of elderly women with sleep disorders and with the susceptibility for falls. Results may be relevant for use in everyday practice and can facilitate future research. Results may also be used to improve the quality of care which is currently provided for patients. The main conclusion is that in elderly women with a lower back dysfuntion, special attention should be paid when prescribing anxiolytics. In general, as our results also suggest, overprescription of medications which affect mental functions in elderly people may be a driving mechanisms for the development of geriatric syndromes and, for this reason, should be avoided and replaced with non-pharmacological interventions.

Ethical approval: All procedures performed in studies involving human participants were in accordance with the ethical standards of the institutional and national research committee and with the 1964 Helsinki declaration and its later amendments or comparable ethical standards.

\section{References:}

Berg,W.P. (1997). Circumstances and consequences of falls in independent community-dwelling older adults. Age Ageing, 26(4), 261-268.

Buffa, R., Floris, G.U., Putzu, P. F., Marini, E. (2011). Body composition variations in ageing. Coll Antropol, 35, 25965.

Buxton, O.M., Cain, S.W., O`Connor, S.P., Porter, J.H., Duffy, J.F., Wamg, W., et al. (2012). Adverse metabolic consequences in humans of prolonged sleep restriction combined with circadian disruption. Science translation medicine, 4, 129ra43.

Cappucio, F.P., Cooper, D., Delia, L., Strazzullo, P., Miller, M.A. (2011). Sleep duration predicts cardiovascular outcomes: a systematic review and meta-analsis of prospective studies. Eur Heart J, 32, 1484-1492.

Cappucio, F.P., D`Elia L., Strazzullo, P., Miller, M.A. (2010). Sleep duration and all-cause mortality: a systematic review and meta-analysis of prospective studies. Sleep, 33, 585-592.

Cruz-Jentoft, A. J., Baeyens, J. P., Bauer, J. M., Boirie, Y., Cederholm, T., Landi, F., et al. (2010). Sarcopenia: European consensus on definition and diagnosis: Report of the European Working Group on Sarcopenia in Older People. Age and ageing, 39, 412-423.

Itani, O., Jike, M., Watanabe, N., Kaneita, Y. (2016). Short sleep duration and health outcomes: a systematic review, meta-analysis and meta-regression. Sleep Medicine, http://dx.doi.org/doi/10.1016/j.sleep.2016.08.006

Kaplan, G.A., Seeman, T.E., Cohen, R.D., Knudsen, L.P., Guralnik, J. (1987). Mortality among the elderly in the Alameda County Study: behavioural and demographic risk factors. Am J Public Health, 77, 307-312.

Koyanagi, A., Garin, N., Olaya, B., Ayuso-Mateos, J.L., Chatterji, S., Leonardi, M., et al. (2014). Chronic conditions and sleep problems among adults aged 50 years or over in nine countries: a multi-country study. PLOS ONE, published online 5 December 2014. http://doi.org/10.1371/journal-pone.0114742

Laird, R.D. (2001). Fall history is an independent predictor of adverse health outcomes and utilization in the elderly. Am J Manag Care, 7(12), 1133-1138.

Leipzig, R.M., Cumming, R.G., Tinetti, M.E. (1999). Drugs and falls in older people: a systematic review and metaanalysis: I. Psychotropic drugs. J Am Geriatr Soc, 47(1), 30-39.

Liu-Ambrose, T. (2009). Dual-task gait performance among community-dwelling senior women: the role of balance confidence and executive functions. J Gerontol A Biol Sci Med Sci, 64(9), 975-982. 
Lord, S.R., Smith, S.T., Menant, J.C. (2010). Vision and falls in older people: risk factors and intervention strategies. Clin Geriatr Med, 26(4), 569-581.

Masud, T., Morris R.O. (2001). Epidemiology of falls. Age Ageing, 30(4), 3-7.

Miner, B., Kryger, M.H. (2016). Sleep in the aging population. Sleep Med Clin. http://dx.doi.org/10.1016/j.jsms.2016.10.008

Montero-Odasso, M. (2009). Dual-tasking and gait in people with mild cognitive impairment. The effect of working memory. BMC Geriatr, 9, 41.

Onder, G., Palmer, K., Navickas, R., Jurevičiene, E., Mammarella, F., Strandzheva M., et al. (2015). Time to face the challenge of multimorbidity. A European perspective from the joint action on chronic diseases and promoting healthy aging across the life cycle (JA-CHRODIS). Eur J Intern Med, 26, 157-159.

Rubenstein, L.Z. (2006). Falls in older people: epidemiology, risk factors and strategies for prevention. Age Ageing, 35(2), ii37-ii41.

Salive, M.E. (2013). Multimorbidity in older adult, Epidemiol Rev, 35, 75-83.

Shan, Z., Ma, H., Xie, M., Yan, P., Guo, Y., Bao, W., et al. (2015). Sleep duration and risk of type 2 diabetes: a metaanalysis of prospective studies. Diabetes care, 38, 529-537.

Sheridan, P.L., Hausdorff, J.M. (2007). The role of higher-level cognitive function in gait: executive dysfunction contributes to fall risk in Alzheimer's disease. Dement Geriatr Cogn Disord, 24(2), 125-137.

Silva, G.E., Goodwin, J.L., Sherrill, D.L., Arnold, J.L., Bootzin, R.R., Smith T., et al. (2007). Relationship between reported and measured sleep times: the sleep heart health study (SHHS). JCSM, 3, 22-30.

Spiegel, K., Tasali, E., Leproult, R., van Cauter, E. (2009). Effects of poor and short sleep on glucose metabolism and obesity risk. Nat Rev Endocrinol, 5, 253-261. http://doi.org./10.1038/nrendo.2009.23

Thomas, K.M., Redd, L.A., Wright, J.D., Hartos, J.L. (2017). Sleep and mental health in the general population of elderly women. J Primary Prevent, published online 26 July 2017. http://doi.org/10.1007/s10935-017-0484-5

Tinetti, M.E. (1994). A multifactorial intervention to reduce the risk of falling among elderly people living in the community. N Engl J Med, 331(13), 821-827.

Tinetti, M.E., Speechley, M., Ginter, S.F. (1988). Risk factors for falls among elderly persons living in the community. New England, Journal of Medicine, 319, 1701-1707.

Tromp, A.M. (2001). Fall-risk screening test: a prospective study on predictors for falls in community-dwelling elderly. J Clin Epidemiol, 54(8), 837-844.

van Iersel, M.B. (2008). Executive functions are associated with gait and balance in community living elderly people. J Gerontol A Biol Sci Med Sci, 63(12), 1344-1349.

van Schoor, N.M. (2002). Different cognitive functions in relation to falls among older persons. Immediate memory as an independent risk factor for falls. J Clin Epidemiol, 55(9), 85562.

Vellas, B.J. (1997). Fear of falling and restriction of mobility in elderly fallers. Age Ageing, 26(3), 189-193.

Wang, Y., Mei, H., Jiang, Y.R., Sun, W.Q., Song, Y.J., Liu, S.J., et al. (2015). Relationship between duration of sleep and hypertension in adults: a meta-analyis. JCSM, 11, 1047-1056.

Winkelman, J.W. (2015). Insomnia disorder. N Engl J Med, 373, 1437-1444.

http://doi.org./10.1056/NEJMcp14122740

Wood, B.H. (2002). Incidence and prediction of falls in Parkinson's disease: a prospective multidisciplinary study. $J$ Neurol Neurosurg Psychiatry, 72(6), 721-725.

Yardley, L., Smith, H. (2002). A prospective study of the relationship between feared consequences of falling and avoidance of activity in community-living older people. Gerontologist, 42(1), 17-23. 